\title{
RursuSpicae
}

1 Rurrsuspicæe Transmission, réception et réécriture de textes, de I'Antiquité au Moyen Âge

1 | 2018

Parodies et pastiches antiques

\section{Rire, parodie et philosophie chez Lucien de} Samosate

Laughter, parody and philosophy in Lucian of Samosata

\section{Brigitte Pérez-Jean}

\section{(2) OpenEdition}

\section{Journals}

Édition électronique

URL : http://journals.openedition.org/rursuspicae/307

DOI : $10.4000 /$ rursuspicae.307

ISSN : 2557-8839

Éditeur :

Université Nice-Sophia Antipolis, IRHT - Institut de recherche et d'histoire des textes

\section{Référence électronique}

Brigitte Pérez-Jean, «Rire, parodie et philosophie chez Lucien de Samosate », RursuSpicae [En ligne], 1 | 2018, mis en ligne le 15 octobre 2018, consulté le 28 avril 2021. URL : http://

journals.openedition.org/rursuspicae/307 ; DOI : https://doi.org/10.4000/rursuspicae.307

Ce document a été généré automatiquement le 28 avril 2021.

RursuSpicae 


\section{Rire, parodie et philosophie chez Lucien de Samosate}

Laughter, parody and philosophy in Lucian of Samosata

Brigitte Pérez-Jean

\section{Mythe, histoire et fiction}

1 Trois termes, mythe, histoire et fiction, doivent être mis en relation à propos de la seconde sophistique et particulièrement à propos de Lucien $^{1}$ pour plusieurs raisons développées par Michel Briand ${ }^{2}$ :

Lucien joue avec ironie, sur lui-même en tant qu'orateur et personnage, et sur les autres auteurs ;

«[...] chaque texte renvoie à d'autres de genre différent (avant-propos, prolalia, récit fantastique, traité de méta-histoire, auto-fiction "; on trouve plusieurs cas où tel texte est repris et comme corrigé par une sorte d'épilogue : Portraits et Défense des portraits, le Pêcheur corrigeant Philosophes à vendre (Vitarum auctio).

[...] le lexique grec relatif à ces notions, non fixé en théorie, autorise les jeux du double-entendre.

2 Michel Briand conclut : « Le $\psi \varepsilon \tilde{v} \delta$ o peut relever du mensonge ou de la fiction, en tant que feintise et le $\mu \tilde{v} \theta$ os peut concerner une tradition autorisée, qui reste à discuter, à prouver, à apprécier et interpréter, comme un monde possible momentané, issu d'une performance. Chez Lucien, mythe, histoire et fiction (en tant qu'auto/méta/transfiction) collaborent à la construction d'une vérité qui n'existe que mise en discours et en scène, suivant ses trois modalités pragmatiques associées au sein d'une même parole, qui tire de cette polyphonie sa cohérence même » (BRIAND, 2010 : 236-237).

3 Ainsi, les questions d'intertextualité interne et externe sont-elles incontournables pour comprendre Lucien ${ }^{3}$. Il est considéré comme l'un des nobles représentants de la dite 
seconde sophistique dont bien des caractéristiques étudiées par la critique peuvent lui être attribuées ${ }^{4}$.

4 On pourra leur ajouter, à partir des récentes études sur le Philopseudès, l'empreinte de la paradoxographie ainsi que l'attrait pour le mime par exemple dans la Danse ${ }^{5}$. Et finalement, sans vouloir nier les différences entre le capitalisme postmoderne et la culture de l'empire romain, on doit admettre avec Tim Whitmarsh leur goût commun pour le spectaculaire ${ }^{6}$. Les textes de Lucien se focalisent de manière obsessionnelle sur la question de la réception de la littérature et de la production artistique.

Par ailleurs, on ne peut aborder Lucien sans avoir à l'esprit la problématique principale de son œuvre, le rapport entre fiction et vérité, problématique qui croise en certains points les notions de récriture et de parodie. La principale leçon des rapprochements que l'on peut faire entre Histoires vraies (HV) et Comment écrire l'histoire? sur la question de savoir comment écrire l'histoire dans l'Antiquité est que Lucien y montre son horreur du mensonge (GEORGIADOU, LARMOUR, 1994: 1448-1507). Du point de vue de l'imagination, on trouvera des rapports étroits entre $H V$ et Icaroménippe, avec le célèbre voyage dans la lune par exemple. Mais la relation entre HV et Comment écrire l'histoire ? à retenir essentiellement pour notre propos est que $H V$ semble apporter le matériel pour la parodie et la critique des historiens qui font du sensationnel, comme si $H V$ illustrait le type d'histoire que Lucien condamne dans l'autre œuvre ${ }^{7}$. On lit donc la fin de la préface de $H V$ comme une parodie des professions de véracité (GEORGIADOU, LARMOUR, $1994: 1506)^{8}$.

6 Lucien s'interroge aussi en philosophe sur le plaisir paradoxal du mensonge dans le Philopseudès, où Tychiadès et Philoclès recherchent à partir de la pensée de Platon, en particulier dans Hippias Mineur, les théories philosophiques sur les mensonges aussi bien que les facteurs psychologiques qui poussent les gens à mentir. À mesure que le dialogue avance et que Tychiadès répète les contes fantastiques des philosophes, Philoclès et lui »expérimentent eux-mêmes l'aspect contagieux des histoires et théorisent leur expérience dans une série de métaphores qui décrivent les effets d'intoxication » (NI-MHEALLAIGH, $2014:$ :22).

\section{Rire et dérision chez Lucien}

7 Sur ce fond de complexe cohérence entre mythe, histoire et fiction, on constatera d'abord que les jeux intellectuels et culturels que les textes de Lucien mettent en œuvre 9 conduisent en principe à des effets comiques et par conséquent, on commencera par s'interroger sur les fonctions du rire que cette œuvre autorise. Nous pourrions proposer quatre hypothèses au rire chez Lucien :

- une fonction critique philosophique: le rire serait un moment dans un processus de réflexion qui supposerait implicitement des thèses et Lucien pourrait passer pour un philosophe au sens classique ${ }^{10}$;

- une fonction nihiliste: le rire entraînerait la destruction totale de toute possibilité de pensée. Et Lucien ne serait même pas un philosophe au sens classique ;

- une fonction esthétique : le plaisir du bon mot l'emporterait sur la question de la vérité avec un Lucien littéraire, un «Lucien écrivain »;

- une fonction existentielle: le rire comme mode de vie neutraliserait l'esprit de sérieux. Lucien devient alors un philosophe existentiel, qui fait l'expérience profonde du rire. 

Byzantins : depuis les termes grecs eironeia, sarkasmos, katagelōs, formes plus raffinées de l'understatement éduqué et élégant, jusqu'aux agressions verbales plus féroces. Les rhéteurs anciens tracent un axe qui irait de l'hilarité jusqu'à la colère, cet axe qui va, selon la sémiotique, de l'euphorie à la dysphorie, comprenant un certain nombre d'étapes qu'on ne saurait détailler, par exemple du skômma au loidorein, etc. (PELLIZER, $2000: 52)$.

11 Ce n'est pas ici le lieu de mener une enquête exhaustive pour savoir si tous les rires analysés tant par les historiens que par les sémioticiens sont représentés chez Lucien : il est évident dès la première lecture qu'on y rit, on y sourit, on y rit jaune, etc. Croisant l'axe historiciste et l'axe rhétorique, on trouvera donc Lucien à la place du satiriste éduqué, et son rire à celle de la dérision sophistico-cynique. Deux autres notions sont également à prendre en considération : d'une part la notion de fantaisie introduite par Bompaire et discutée par MacLeod, en laissant de côté tous les débats sur la mimesis et, d'autre part, la formule de spoudogeloios qui semble bien caractériser Lucien dont R. Bracht Branham donne une explication éclairante : "There is no serious Lucian who merely uses humor incidentally or who simply happens to amuse an audience in addition to doing something serious " (BRANHAM, 1989 :7-8). C'est pourquoi l'auteur utilise tout au long de son étude le terme de serio-comic. Pour être plus précis, il faudrait enfin pouvoir comparer les divers classements des œuvres par genres, par exemple chez Nesselrath dans Die Neue Pauly et les classements par sous-genres comiques, par exemple chez R. B. Branham.

\section{Les traditions savantes et les fausses valeurs}

À partir de cette approche du serio-comic lucianesque, je m'interrogerai plus précisément sur ses ressorts, afin de comprendre leur fonctionnement dans la relation avec les traditions savantes antérieures, textuelles et/ou doxographiques, en privilégiant les œuvres de Lucien qui prennent pour cibles les philosophes et la philosophie. Nombreuses sont les références à la poésie homérique et aux comédies d'Aristophane qui ont été relevées par les commentateurs et variées sont les victimes de la moquerie de Lucien : les vivants et les morts, les dieux, les femmes, les tyrans, la pédérastie, les athlètes, les faux prophètes, les devins, les chrétiens, les vieillards ; les philosophes semblent être privilégiés. Lorsqu'il parle des philosophes, l'auteur franchit la frontière du sourire fin et élégant, du rire heureux et relaxant, pour atteindre la 
moquerie, l'insulte, la vexation, comme on le lit dans le Peudologiste et le Banquet ou les Lapithes -et bien sûr La mort de Pérégrinos.

Lucien ou son « masque » (DUBEL, 1994) engage le lecteur ou l'interlocuteur à rire, comme en témoignent les nombreuses occurrences du terme geloion ( risible» ou « ridicule ») dans les Opuscules, et le rire des personnages ou des interlocuteurs du narrateur joue avec celui du lecteur: Karen Ni-Mheallaigh analyse les liens entre lecteur, personnage et auteur (sans oublier les doubles de Lucien) mais s'attache surtout au reader Tychiadès dans le Philopseudès (vs Philopseudeis), un opuscule qui apparaît plus fort que les Histoires vraies pour comprendre les idées antiques sur la lecture de fiction. Eucratès y est un lecteur philosophe tandis que les autres, dont Tychiadès, sont des lecteurs de paradoxa (NI-MHEALlAIGH, 2014). Il se produit donc dans chacune des scènes un jeu complexe entre fantaisie, imaginaire et réalité ${ }^{11}$ qu'on ne saurait réduire à un jeu parodique à partir de textes ou de théories philosophiques.

Les anthropologues analysent les ressorts du rire et de la dérision comme une « série de messages de dominance et/ou de menace qui se transforment par inversion subite en bruyant démenti d'une prétention impossible » (PELLIZER, 2000: 49): la lumière se fait soudain sur la faiblesse ou l'infériorité du prétendant, considéré comme potentiellement hostile en raison de son appartenance à telle catégorie sociale ou ethnique "autre» que celle des rieurs. Or, si l'on remplace la menace primaire d'agression physique de la part d'un Hercule de foire par «la crainte de la confrontation intellectuelle, des preuves d'intelligence, de ruse, de culture, on décode alors sans peine, sur le mode d'un mécanisme de défense, toutes les histoires qu'on raconte pour couvrir de ridicule l'autre » (PELLIZER, $2000: 50$ ). Je voudrais donc montrer comment, s'agissant de la philosophie et des philosophes, Lucien joue de ce principe de confrontation intellectuelle et païdeutique aussi bien dans le domaine pratique que dans la récriture des théories. En effet, dans la période de la philosophie dite médioplatonicienne, c'est-à-dire dans l'ère culturelle de la seconde sophistique, les jeux et les détournements ne se limitent pas à des effets textuels, mais s'intéressent particulièrement dans l'esprit de la philosophie sérieuse à la question du bios.

L'un des fonctionnements du principe de confrontation a été mis en lumière par Alain Billault ${ }^{12}$ à propos du Pseudologiste, dont le personnage titre est un adversaire (Adrien de Tyr ?) contre lequel Lucien porte des accusations pleines de sous-entendus concernant son passé et spécialement ses rapports avec l'argent et avec le sexe, renversant ainsi les codes du genre biographique. "Montrer le vrai visage d'un homme en remontant à ses débuts dans la vie et en retraçant ses mauvaises actions qui restent ignorées de ses admirateurs, Lucien le fait aussi dans Alexandre ou le faux prophète, dans la Mort de Pérégrinos et dans le Maître de Rhétorique » (BILlault, 1994, p. 120). ${ }^{13}$

La quintessence du principe de la prétention impossible, c'est l'opposition entre la barbe du philosophe et l'absence de sagesse, remarque inlassablement ressassée par Lucien, ou bien entre la qualité de philosophe et l'intempérance alimentaire et alcoolique dans le Banquet ou les Lapithes, ou tout simplement dans le dialogue significatif où éclate la contradiction dissimulée dans la barbe qu'il faut couper à la hache, échange entre « le philosophe » et Ménippe (Dialogue des morts 10.10). 
Quelques types d'écart correspondent à la découverte de l'infériorité de tel ou tel prétendant à une supériorité, certains de ces écarts étant propres aux philosophes.

- L'inculture : le meilleur exemple de confrontation entre agnoia et paideia est le bibliophile ignorant ${ }^{14}$.

- Les fausses valeurs, au premier chef l'argent et le pouvoir : les tyrans et les riches ont soif de puissance et de richesses.

- La prétention des philosophes non à la détention de la vérité mais à la célébrité ; c'est ce point qui froisse l'auteur à propos de Pérégrinos ${ }^{15}$.

- Pour les philosophes surtout, l'écart entre les paroles et les actes, comme en témoigne Pérégrinos encore ${ }^{16}$ :

Alors que nous étions surpris, de nuit, en pleine mer Égée, une tornade s'étant élevée soulevant une immense vague, il geignait avec les femmes, lui cet homme admirable qui semblait plus fort que la mort. (De morte Peregrini, 43)

On peut légitimement penser que cette anecdote renvoie à celle qui montrera (chez Diogène Laërce) Pyrrhon imperturbable dans la tempête ${ }^{17}$.

Enfin, la tension principale qui révèle soudain l'écart entre la prétention et la faiblesse est évidemment le passage de la vie à la mort, sujet de nombreux dialogues: les Dialogues des morts et d'autres mises en scène de puissants arrivant aux Enfers sont d'une remarquable efficacité à cet égard, par exemple le Tyran. Les prétentions impossibles se révèlent donc davantage chez les morts que chez les vivants et comme le dit Dialogues des morts I, Ménippe, s'il a bien ri sur terre, doit venir aux enfers pour y rire davantage. Le ressort principal de la relation entre le rire et la mort est-il pour autant une forme de moralisme de Lucien comme on peut le penser pour Dion et Plutarque $^{18}$ ? Certains critiques en doutent, dans l'idée que Lucien ne propose aucun redressement (BRANHAM, 1989 ; NI-MHEALLAIGH, 2014) ${ }^{19}$.

\section{Lucien philosophe?}

Qu'il rit ou fait rire des philosophes, du type du philosophe, chacun en conviendra. Qu'il philosophe en riant ou rit en philosophant, c'est lui qui l'affirme dans une célèbre formule du Bis accusatus. Mais Lucien est-il un philosophe ${ }^{20}$ ?

21 La lecture de la Critique de la raison cynique de Peter Sloterdijk ${ }^{21}$, parue deux cents ans après la Critique de la raison pure, nous donne l'assurance que Lucien était un vrai philosophe. Le livre de Sloterdijk qui concerne surtout les philosophes allemands du $\mathrm{XIX}^{\mathrm{e}}$ siècle et du $\mathrm{XX}^{\mathrm{e}}$, le cynisme versus l'Aufklärung, est considéré comme relevant du néo-nietzschéisme suivant les traces du néo-cynisme de Nietzsche lui-même (NIEHuEs PRÖBSTING 1996). Il est à compléter par les remarques de Foucault dans son cours au collège de France désormais publié sous le titre Le courage de la vérité (FOUCAULT 2009: $154-157$;177-190). Selon Sloterdijk, il y a bientôt trente ans, notre époque, revenue des illusions de notre rationalisme, est ébranlée dans sa croyance en l'Aufklärung: la conviction que le mal résulte de l'ignorance et qu'il suffit de savoir pour le guérir. Le cynisme, aujourd'hui phénomène universel, est la réponse à cette désillusion.

Comme remède et comme dépassement, l'auteur suggère de redécouvrir les vertus du cynisme antique (ou, plus exactement, du kunisme) que pratiquait le philosophe de Sinope : le rire, l'invective, les attaques. Ce terme « kunisme » est la traduction adoptée pour le terme allemand Kynismus que H. Niehues Pröbsting a proposé d'opposer au 
terme Zynismus, distinction que Sloterdijk a reprise (NIEHUES PRöBSTING 1988). La redécouverte de la version antique du cynisme pourrait renouveler la chance de l'Aufklärung dont le projet le plus intime est de transformer l'être (Sein) par l'être conscient (Bewusstsein).

«Là où le cynique sourit avec mélancolie et mépris, du haut de sa puissance et de sa désillusion, la caractéristique du kunique est de rire fort et sans gêne, au point que les gens distingués secouent la tête. Le rire du kunique vient des entrailles, il a un fond animal et se manifeste sans inhibition. L'homme qui prétend être réaliste devrait pouvoir rire de la sorte, de ce rire total et décrispant qui fait table rase des illusions et des poses " (SLOTERDIJK, 1987: 188). Ce rire est aussi bien celui de Diogène que de " maints Bouddhas riants » : il " a quelque chose de ce rire d'entrailles animal, en même temps extatique et réaliste, qui par ses bonds et ses secousses s'oublie tellement qu'il n'y a plus de moi en lui qui rit, mais seulement une énergie sereine qui s'autocélèbre » (SLOTERDIJK, 1987 : 188-189).

Dans le chapitre Cabinet des Cyniques de Sloterdijk, seuls deux membres sont historiques, Diogène de Sinope et Lucien : Lucien le moqueur ou la critique change de camp (SLOTERDIJK, 1987 : 219). Selon l'auteur, le kunisme de l'époque impériale a pu être caractérisé comme le mouvement hippie et marginal de l'Antiquité. L'énorme succès de sectes philosophiques comme des nouveaux cultes religieux originaires de l'Asie mineure ${ }^{22}$ montre que le besoin "d'auto-affirmation individualiste" s'est fait sentir. "L'individualisme paléo-humaniste et noble des Romains cultivés regardait avec mépris l'agitation des nouveaux individualistes qui se comportaient en partie en asociaux» (SLOTERDIJK, 1987:225). Lucien, en tant que cultivé et conservateur, représente pour le philosophe le nouvel accent cynique qu'adoptent les intellectuels des époques avancées dès qu'on provoque leur mépris.

Puisque l'Antiquité, particulièrement à l'époque du haut Empire, classait nécessairement les philosophes par sectes, il n'apparaît pas déplacé de se demander si Lucien peut être rattaché effectivement à une secte philosophique. Il n'est plus aussi aisé que ce l'était il y a une trentaine d'années de recourir à l'étiquette d'éclectique dans la mesure où ce terme désigne proprement certains philosophes comme Polémon. Pourtant, Lucien écrit de Démonax, ce philosophe qu'il dit avoir fréquenté et dont il écrit la vie dans un texte diatribique que $C$. P. Jones a qualifié d'" autobiographie indirecte »: « il ne se borna pas à un type de philosophie; mais ayant fait une synthèse de plusieurs philosophies, il se gardait bien de révéler celle d'entre elles qui avait sa faveur " (Vie de Démonax 5$)^{23}$.

Mon hypothèse a toutefois été, depuis la lecture de Sloterdijk, que s'il le revendiquait comme témoin privilégié de son kunisme, c'est que Lucien était cynique. La publication du cours de Foucault au Collège de France confirme l'hypothèse; si elle est fausse, il restera toujours l'étiquette partout répétée, à propos de Lucien ou d'autres, de Halbphilosoph.

\section{Les cibles philosophiques de Lucien}

Quoi qu'il en soit, Lucien est un sophistès de son temps qui possède une culture philosophique certaine. De Platon il ne connaît pas seulement le Timée, le texte-clé pour les platoniciens de son époque, mais son Philopseudès dont le sous-titre est Apistia est à 
mettre en rapport avec le traitement de ce thème dans les Lois (NI-MHEALLAIGH, 2014: 79-80), son Banquet ou les Lapithes montre qu'il connait Euthydème, le Banquet bien sûr et le Phèdre de l'intérieur puisque, par exemple, le début parodie le jeu entre Phèdre et Socrate concernant l'amour des logoi ${ }^{24}$. Par ailleurs, même si Lucien possède un stock de plaisanteries qu'il ressert à l'envi ${ }^{25}$, associant un philosophe à un détail vestimentaire, physique ou même théorique, il dit parfois davantage de Démocrite que son rire, d'Héraclite que ses pleurs, de Chrysippe que sa calvitie, de Socrate que ses ideai...

S'il a une orientation philosophique, peut-on la découvrir en fonction de la satire ou de la parodie des philosophes présentes dans de nombreux ouvrages, dialogiques ou non dialogiques? Quelles sont ses cibles chez les philosophes?

Il semble en effet différent de se moquer de tel philosophe, même s'il représente une secte ou et de s'attaquer à l'ensemble des philosophes. Se moquer de l'ensemble de la profession, c'est en réalité à travers les personnages qui représentent chaque secte opposer des opinions et donc emprunter le chemin du scepticisme, mais l'emprunter seulement. La diaphônia au lieu de conduire à la suspension du jugement doit conduire au rire. C'est le résultat de l'entretien entre Hermotime et Lycianos sur la diversité des sectes et de leurs théories qui demanderaient plus de deux cents ans pour être toutes étudiées, même si Ménippe en conclut à l'incertitude universelle ${ }^{26}$.

La position de Lucien n'est pas non plus un relativisme. Chez lui, l'accumulation des opinions différentes est l'une des clés du comique, l'accumulation étant un des traits de son style. Lucien n'est pas un adepte du scepticisme philosophique, même s'il connait la version néo-pyrrhonienne du scepticisme, telle qu'elle nous sera transmise par Sextus Empiricus, comme en témoigne l'Hermotime avec le trope de reductio ad infinitum d'Agrippa (HANKInson 1995: 151); d'autres textes s'amusent de la formule de l'akatalèpsia, par exemple les Philosophes à vendre, où Pyrrhon lui-même semble moqué sous le nom de Pyrrhias (le Rouquin) ${ }^{27}$.

Enfin, dans les œuvres où les sectes ne sont pas toutes représentées, Lucien fait des associations, par exemple Stoïciens vs Epicuriens, tantôt réunit les grandes écoles représentées à Athènes sous protection impériale, par deux ou par trois, tantôt ajoute des présocratiques, Empédocle et Pythagore le plus souvent. Ou encore comme dans le deuxième livre d'HV, il distingue les philosophes admis ou pas sur l'île des bienheureux $(H V, 17-18)$.

\section{L'écart de la parodie : maîtres ou disciples ? passé ou présent?}

Dans Philosophes à vendre (Vitarum auctio) une ambiguïté apparente affecte la question de savoir qui est mis à la vente? Pyrrhon ou bien un/le pyrrhonien sous le nom de Pyrrhias? Chrysippe ou le stoïcien? En réalité, les philosophes y sont bien individualisés, et précisément le dialogue suivant, écrit, semble-t-il, en même temps, Piscator (le Pêcheur ou les Ressuscités), donne la parole aux philosophes ressuscités qui prétendent que Parrhèsiadès (Lucien) doit être condamné pour le contenu du dialogue précédent; il se défend alors devant le tribunal présidé par Philosophie en plaidant qu'il ne s'y agissait pas d'eux, mais des représentants actuels de leurs écoles respectives qui sont des pseudo-philosophes, comme le montrera la deuxième partie du texte avec la pêche aux groupes de philosophes du haut de l'Acropole (Piscator 42). 
illeurs, on ne peut pas voir dans la contradiction entre les différents philosophes, ou mieux entre les différentes vies philosophiques de la Biôn prasis, le ressort principal de la satire. La situation dramatique est, certes, amusante, avec le décor d'une vente aux enchères, les personnages du crieur, des acheteurs et le défilé des philosophes sur l'estrade. Mais la finesse de la parodie vient de la connaissance précise des systèmes philosophiques mis à l'étalage, dans l'argument de vente énoncé par le crieur, et de l'écart entre cette précision scientifique exprimée avec les termes philosophiques appropriés et l'incapacité de l'acheteur à se représenter de quoi il peut s'agir ainsi que l'utilité didactique qu'il pourrait retirer de son éventuel achat. C'est pourquoi certains articles restent invendus; d'autres sont acquis, mais à des prix très différents; c'est parce que Diogène aura été soldé à deux oboles qu'il sera le premier à accuser Parrhèsiadès dans le deuxième dialogue. Le lecteur d'aujourd'hui doit donc connaître la doxographie philosophique antique ou avoir à disposition Diogène Laërce, Sextus Empiricus, le Didaskalikos, le Long et Sedley et le Diels-Kranz, ou encore le récent volume de Laks et Most (Laks, Most ${ }^{2016)}$ pour apprécier tout le sel parodique des interrogatoires subis par les divers philosophes.

Or les questions de l'acheteur ne concernent pas uniquement des anecdotes sur tel ou tel philosophe, des récits ou des topoi comme ceux que Lucien aime à citer, par exemple le plongeon d'Empédocle dans l'Etna ou la cuisse d'or de Pythagore, le rire de Démocrite opposé aux pleurs d'Héraclite. Les thèses philosophiques évoquées ne concernent pas uniquement, comme on pourrait le penser, l'éthique ; on trouve aussi les atomes et le vide de Démocrite, les idées de Platon, les syllogismes de Chrysippe, le flux héraclitéen... En revanche, la qualification donnée par le « commissaire priseur » à chaque présentation d'un philosophe par un qualificatif, souvent un superlatif différent renvoie, de toute évidence, aux éthiques médio-platonicienne ou aristotélicienne du deuxième siècle, comme le montrent ces extraits de Philosophes à vendre.

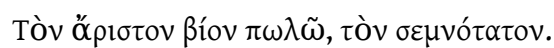

Je mets en vente la meilleure vie, la plus respectable. (Pythagore)

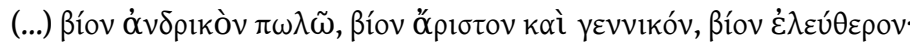

(...) je vends une vie mâle, une vie excellente et courageuse, une vie libre. (Diogène)

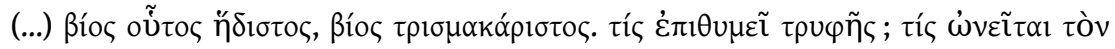

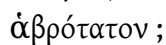

(...) voici une vie vraiment délicieuse, et trois fois heureuse. Qui désire une vie de plaisir ? Qui achète la vie la plus délicate? (Aristippe)

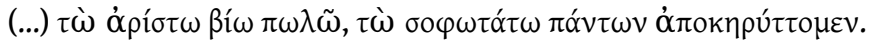

(...) je vends les deux meilleures vies. Nous mettons aux enchères les deux plus sages au monde. (Héraclite et Démocrite)

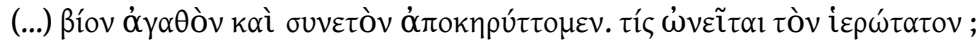

C'est une vie honnête et intelligente que nous vendons. Qui achète la plus sainte des vies? (Socrate)

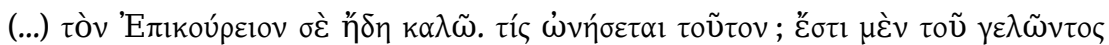

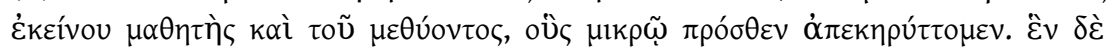

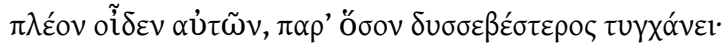

À toi maintenant l'Épicurien, je t'appelle. Qui l'achètera? C'est le disciple de ce rieur et de l'ivrogne que nous avons mis en vente tout à l'heure. Il sait une chose de plus qu'eux, dans la mesure où il est plus impie. 


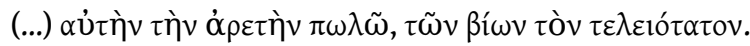

(...) je vends la vertu même, la vie la plus parfaite. (Chrysippe)

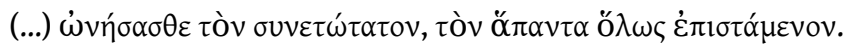

Achetez le plus intelligent, celui qui sait tout. (Le péripatéticien)

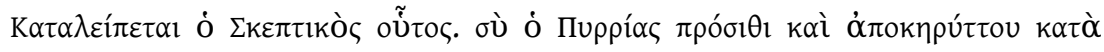

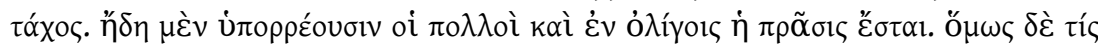

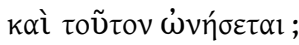

Il reste ce sceptique. Toi, Pyrrhias, approche qu'on te vende au plus vite. Car la foule commence à s'écouler et la vente va se passer en petit comité. Qui alors achètera aussi notre homme?

À la décharge de Parrhésiadès-Lucien, quand il prétend pour sa défense qu'il n'attaquait pas les grands maîtres du passé, on remarquera qu'Épicure est de fait représenté sur ce marché par un épicurien. En réalité, on trouve autant d'Épicures que d'épicuriens dans le corpus de Lucien.

Dans le Banquet, au contraire, les philosophes représentent les différentes sectes mais sont bien des personnages contemporains de Lucien, voire d'authentiques philosophes. Toutefois, ils ne font montre d'aucun goût pour une joute théorique, puisque le dialogue est un renversement du Banquet platonicien (R OMERI 2002). Ils sont donc moqués en tant que groupe, et de surcroit non pas tant pour leurs contradictions que parce qu'ils sont ivrognes et goinfres et qu'ils iront jusqu'à se molester pour une cuisse de poulet.

Dans Le pêcheur ou les ressuscités, voilà les grands philosophes de l'Antiquité revenus sur terre et nous sommes en présence des modèles et non des copies : même Platon et Aristote sont présents. Pourquoi se référer aux philosophes du passé ? On ne saurait suivre Bompaire lorsqu'il pose "l'actualité des philosophes contre la tradition des rhéteurs » à propos du Pseudologiste. D'un côté, la tendance de la philosophie à cette époque (que ce soit platonisme ou aristotélisme, stoïcisme ou épicurisme) consiste, dans les appellations au moins, à se référer aux fondateurs des sectes devenues depuis le premier siècle l'expression même de la philosophie; de l'autre, la mode des biographies met sur le marché littéraire ou celui de la dite "philosophie populaire " des personnages comme Pythagore dans la wonder-literature, comme Diogène dans les exercices rhétoriques ou Socrate chez les socratiques de toute obédience.

Lucien répond au début de la Vie de Démonax (2) que les philosophes du passé sont en quelque sorte «au programme » : « il est juste de parler de lui pour que son souvenir subsiste dans l'élite, autant qu'il dépend de moi, et pour que les jeunes gens les plus doués, qui se tournent vers la philosophie, puissent ne pas se régler sur les seuls modèles anciens et se proposer un canon qui soit bien de notre temps et imiter cet homme qui fut le meilleur des philosophes connus de moi ${ }^{28}$.

\section{Lucien cynique?}

Les Cyniques sont souvent ménagés par rapport aux autres sectes comme dans les Dialogues des morts ${ }^{29}$ mais parfois eux aussi, ou surtout, comme Diogène, sont la cible des moqueries de Lucien; la figure récurrente du philosophe barbu avec sa besace et son bâton ne saurait correspondre à un Aristippe parfumé et c'est donc bien Diogène qui est 
moqué comme archétype du philosophe. Diogène, on l'a vu, est associé aux autres " philosophes à vendre " (il est le deuxième candidat, sans respect de chronologie) et le marché ne lui attribue la valeur que de deux oboles. Il est bien évidemment dans l'histoire de la philosophie le modèle du philosophe cynique et a eu une influence considérable chez des auteurs comme Epictète, Dion et Julien; comme on le lit au livre VI de Diogène Laërce, ses positions apparaissent parfois comme teintées de stoïcisme (Ariston de Chio). Or, outre le riche matériel transmis par Diogène Laërce, Diogène est aussi l'objet de la moquerie de toute une littérature rhétorique, en particulier dans les chries propres à l'éducation rhétorique qui collectent des centaines - on dit même un millier- de dits et d'anecdotes concernant Diogène. Il n'est donc pas étonnant que tout le folklore cynique autour de Diogène et particulièrement son anaideia (impudeur) apparaissent clairement comme objets de moquerie chez Lucien : «Fais hardiment sous les yeux de tout le monde ce qu'on aurait honte de faire tout seul et, dans les plaisirs sexuels, choisis les plus ridicules (geloiotera). » (Vitarum auctio, 10).

Par ailleurs, Lucien ne ménage pas ses critiques contre les cyniques de son temps : le célèbre texte sur la mort de Pérégrinos parait tellement hostile à la secte que certains critiques ont pu douter de l'authenticité de la Vie de Démonax en raison de la déférence de Lucien pour ce cynique. On y a vu une contradiction. Lucien veut-il brouiller les pistes? On peut répondre à cette contradiction en opposant simplement les titres Vie de Démonax vs Mort de Pérégrinos.

41 Ménippe est évidemment un cas à part : il est le modèle de Lucien et son porte-parole, même s'il existe un débat chez les commentateurs pour savoir jusqu'où porte l'« ombre » de Ménippe (віLlault 1994). Quoi qu'il en soit, on ne sait presque rien de Ménippe, mais les textes qui lui sont attribués portent en effet des titres comparables à ceux de Lucien.

\section{Lucien sceptique ? Lucien épicurien?}

Face à la question de l'orientation philosophique de Lucien, les commentaires nous parlent traditionnellement d'éclectisme, d'épicurisme mâtiné de scepticisme ${ }^{30}$ et l'on peut rappeler que la tradition savante, française au moins, a longtemps classé Lucien plutôt parmi les épicuriens ${ }^{31}$, en lien évident avec la question de l'anthropomorphisme des dieux, des sacrifices; mais n'oublions pas que ce sont des thèmes tout à fait identifiés dans le cynisme originel (GOULET-CAZÉ 1996). Sur la question du scepticisme, les commentateurs s'accordent sur le fait que connaître le scepticisme n'entraîne pas une adhésion à la conduite de vie sceptique (BONAZZI 2008 et MESTRE 2013) et il semble que la voie sceptique soit surtout pour Lucien un moyen facile de renvoyer dos à dos les dogmatiques, avec l'argument de la diaphônia comme on l'a vu. Pour être sceptique, il faut faire un pas de plus vers l'époché. Affirmer que Lucien «ne prétend point voir la vérité » (MESTRE 2013 : 80) ne fait pas en effet de lui un adepte du scepticisme.

43 À la fin de l'Alexandre, Lucien ou le narrateur qui s'adresse à Celse l'épicurien dit qu'il "voulait surtout venger Épicure, cet homme vraiment sacré, ce génie divin qui seul a connu réellement les belles choses et transmis ses connaissances à ses disciples dont il a été le libérateur » en démystifiant l'oracle d'Alexandre le faux prophète. Les liens les plus nets avec l'épicurisme apparaissent dans les deux pseudo-biographies que sont Alexandre et la Mort de Pérégrinos. Ce dernier texte, avec un tel titre, apparaît comme un 
pastiche de biographie (BRANHAM 1989: 185-200). L'intérêt manifeste de Lucien pour l'épicurisme tient dans sa capacité à démystifier les illusions de la croyance ; on ne peut pour autant le dire épicurien. Je m'en tiendrais volontiers à la formule de Philippe Renault dans l'introduction à la traduction de la Nekyomancie : « la pensée cynique, dont Lucien est un adepte, même s'il la mâtine généralement avec les idées épicuriennes qui ont également sa prédilection $»^{32}$.

\section{Les adversaires de Lucien}

doctrine sont présentés dans une réduction à l'absurde et n'intéressent pas vraimen Lucien. Si les évocations des philosophes anciens ne sont que « la répétition banale des lieux communs les plus superficiels » (MESTRE 2013 : 66), on lui a attribué en revanche un réel intérêt pour Platon en raison d'abord de son intérêt pour la forme dialogue. «L'utilisation du dialogue pour faire la satire de ses contemporains montre déjà, à mon avis, une adhésion à la tradition philosophique des anciens et, surtout, à Platon » écrit F. Mestre. Elle considère que bien connaître les Dialogues de Platon ne fait pas de Lucien un platonicien convaincu, au contraire; " en effet, tandis que la satire qu'il adresse au Platon philosophe est très dure, Lucien a l'air d'être très à l'aise dans le Platon littéraire, et se sert très souvent de l'œuvre platonicienne pour y installer sa parodie » (MESTRE 2013 : 66). Ainsi la mimésis du dialogue socratique et des discussions de banquet sont une partie fondamentale de ce que Reardon appelait sa "création rhétorique ». (MESTRE 2013 : 80). Enfin, dans l'analyse des Histoires vraies, l'auteure montre comment ce texte exprime les difficultés pour dire le vrai à travers la fiction et considère que "l'allégorie platonicienne est à la base de ses récits fantastiques » (MESTRE $2013: 80$ ).

C'est trop opposer, selon moi, un Lucien «créateur rhétorique et littéraire exceptionnel » au Lucien philosophe et l'on doit se demander s'il faut attribuer à Lucien, au delà de la satire des banquets de philosophes, une critique du platonisme traditionnel, en particulier parce qu'il serait un trait de la seconde sophistique dans l'esprit des discours Прò $\Pi \lambda \alpha \alpha ́ \tau \omega v \alpha$ d'Aelius Aristide ? Ce n'est pas le cas et en réalité les accusations comiques contre Platon sont d'ordre politique, en relation avec ses fréquentations du tyran de Syracuse ${ }^{34}$.

\section{Et Socrate?}

48 On se trouve en général, chez Lucien comme ailleurs, confronté à une confusion Socrate/Platon comme dans l'allusion à la kallipolis de la République dans laquelle Socrate se tient renfermé à l'abri, ou dans la tirade d'Ion dans le Banquet en faveur de la 
communauté des femmes, et surtout dans le choix attribué à tous les deux de la paiderastia $^{35}$, tirade où l'on appréciera les marques de parodie du dialogue platonicien. C'est avant tout cette tendance amoureuse qui caractérise le personnage lucianesque de Socrate, comme en témoigne dans le sixième Dialogue des morts l'allusion à Charmide et Phèdre.

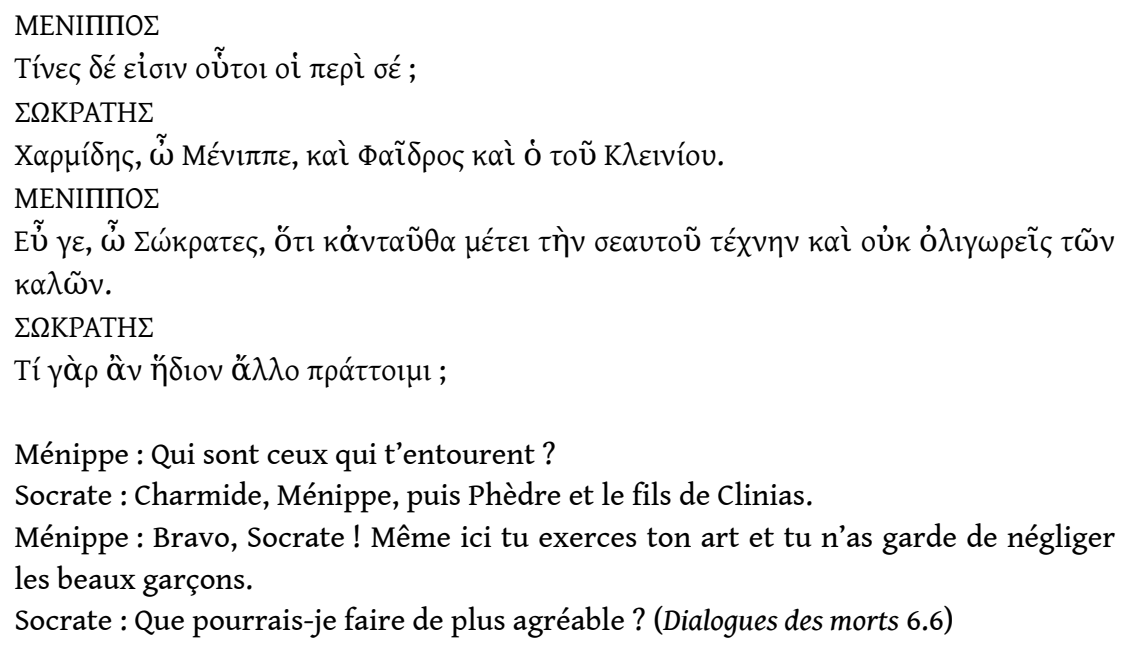

La plaisanterie type sur ce sujet tourne autour de la couverture sous laquelle Alcibiade voudrait se glisser avec Socrate, en relation avec la fin du Banquet de Platon. Suffit-elle à caractériser le Socrate de Lucien? L'image de Socrate à l'époque impériale est héritière d'une longue histoire de la controverse entre des écoles rivales, qui se prolongea pendant toute l'époque hellénistique (CAMPOS DAROCA $2016: 67-84)^{36}$. Lucien ne semble pas participer à la controverse, n'est pas pas sensible au caractère risible du Socrate platonicien et fait déclarer à l'un de ses personnages qu'il n'aime pas l'ironie socratique ; à l'issue d'une enquête trop rapide, on peut conclure qu'il se moque surtout de son amour des jeunes gens, mais la question de la paiderastia ne saurait se réduire à une simple plaisanterie à caractère sexuel. Les questions sur l'érotique de Socrate étaient sérieusement envisagées par les philosophes de ce temps comme en témoignent les Dialexeis XVIII-XXI de Maxime de Tyr ${ }^{37}$. Dans la conférence XVIII, Maxime paraît faire face à de nouveaux accusateurs de Socrate, scandalisés, semble-t-il, par ses propos amoureux. Lucien en était-il scandalisé ?

En tout cas, Démonax, le « bon cynique » semble bien révérer Socrate :

Interrogé un jour sur lequel des philosophes avait sa faveur, il dit: Tous sont admirables : je révère Socrate, j'admire Diogène et j'aime Aristippe ${ }^{38}$ (Vie de Démonax 67).

51 Au contraire, Théagène, le disciple dément et fanatisé de Pérégrinos, le mauvais cynique, est accusé de placer son maître au-dessus de Diogène et d'Antisthène et même de Socrate, ce qui paraît scandaleux à l'auditeur/narrateur ${ }^{39}$.

\section{Philosophes ou collègues?}

Il faut enfin constater que, même si les philosophes occupent une place de choix parmi les cibles diverses de Lucien, bien souvent le groupe des philosophes fait partie d'un ensemble plus vaste, les pepaideumenoi, en association étroite avec les gens de lettres,

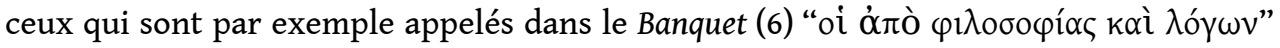
(les spécialistes de la philosophie et du langage...). On retrouve ici la difficulté à définir 
ce qu'est un philosophe pour les auteurs de la seconde sophistique ${ }^{40}$. Et par ailleurs, comme on l'a vu, Lucien ne vise pas tant les auteurs des grands systèmes philosophiques du passé ou leurs représentants (leurs followers) que tout simplement ses « collègues ».

Mettons un terme à ce faux suspense : la philosophie de Lucien est bien proche de celle de Démonax. Michel Foucault insiste sur le fait que la Vie de Démonax est un éloge, celui du bon cynique (Foucault 2009 : 156-7). Démonax est l'homme de la parrhésia ${ }^{41}$ et de la philanthropia ${ }^{42}$. Selon Foucault, dans le mode de vie du cynique, la parrhésia n'est qu'un élément avec le bâton, la besace, la pauvreté, l'errance, la mendicité dont il développe les fonctions dans la suite du cours, mais un élément fondamental pour son «courage de la vérité ». Or ce ne sont pas ces éléments-là du mode de vie cynique qui permettent de rapprocher Lucien de Démonax, mais bien (avec la parrhésia) son humour, en particulier ses bons mots. On pourra citer « le péripatéticien boiteux » ou le jeu de mots sur hyperon/Hypéride ${ }^{43}$ et surtout la succession de véritables chries, comme celle du sophiste hermaphrodite Favorinos d'Arles.

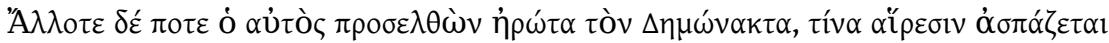

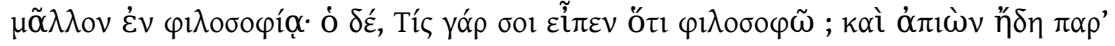

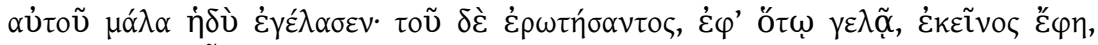

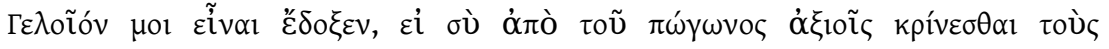

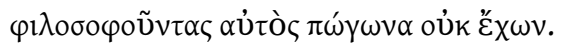

Une autre fois le même Favorinos venait demander à Démonax quelle école il préférait en philosophie. Il répartit : « Qui donc t'a dit que je suis philosophe ? » Sur ce, il le quittait en riant de bon cœur, mais Favorinos lui demanda pourquoi il riait. Il répondit: «Il m’a semblé drôle que tu prétendisses distinguer ceux qui pratiquent la philosophie d'après leur barbe, toi qui n'as pas de barbe!» (Vie de Démonax 13).

On appréciera particulièrement la réponse faite au sophiste vantard par Démonax, qui est doux et ne mord pas :

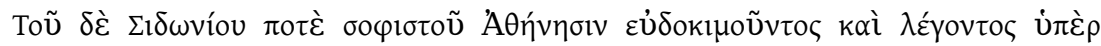

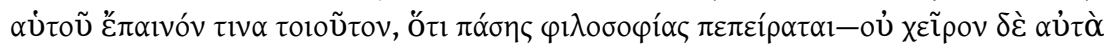

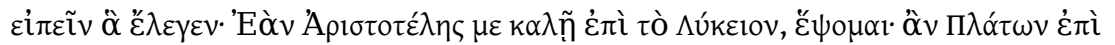

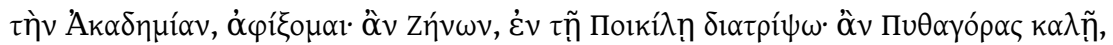

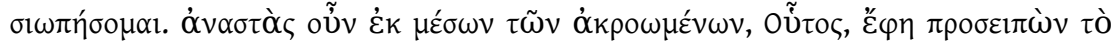

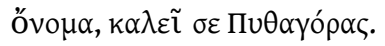

Un jour le sophiste de Sidon, qui était en vogue à Athènes, prononçait son propre éloge à peu près sur ce thème: il connaissait d'expérience la totalité de la philosophie. Mais il vaut mieux rapporter ses propres paroles: «Si Aristote m'appelle au Lycée, je le suivrai ; si c'est Platon à l'Académie, je m'y rendrai ; si c'est Zénon, je passerai mon temps au Portique; si c'est Pythagore, j'observerai le silence. » Alors Démonax se leva au milieu de l'auditoire: «Hé toi !, dit-il, en le désignant par son nom, Pythagore t'appelle ». (Vie de Démonax, 14)

\section{Conclusion}

Étudiant le vocabulaire du rire chez Aristophane (rire de moquerie, rire provoqué, rire paradoxal), A. Sommerstein renvoie à la notion exposée par R. J. Hankinson de « rire du plaisir commun » qui ne cause aucune honte ou douleur et dont il analyse les sources chez Aristophane; il propose donc de distinguer entre "rire de» et "rire avec» (SOMMERSTEIN 2000 : 71-73). 

intellectuels des époques avancées dès qu'on provoque leur mépris. C'est donc ici Pérégrinos-Protée qui provoque le mépris de Lucien par son suicide tragique et héroïque pour satisfaire sa propre aspiration à la gloire. De fait, sloterdijk finit par exclure Lucien de son cabinet idéal des cyniques, parce que « le rire de Lucien est trop strident pour être gai ; il atteste plus de haine que de souveraineté ». Lucien demeure pour lui « l'ironiste hautement cultivé » dont l'existence est remise en cause par « les kuniques qui attaquent l'État romain, la civilisation hellénique et la psychologie des bourgeois ambitieux aimant le confort » (SLOTERDIJK 1987 : 226). Le philosophe entend par «la critique change de camp» le fait que le cynisme passe de l'insolence d'un Diogène à l'« idéologie cynique » qu'il attribue à Lucien : « Son criticisme est devenu opportunisme; il entend tirer parti de l'ironie des puissants qui voulaient se moquer des critiques existentiels qui s'en prenaient à eux » (SLOTERDIJK 1987 : 225).

Pour ma part, en raison du lien étroit que l'on peut deviner entre Lucien et le bon cynique Démonax, j'attribuerais volontiers à « l'ironiste hautement cultivé » la capacité d'un rire partagé, inclusif au sens de Sommerstein.

60 Les accents ironiques et parodiques des textes de Lucien mettant en scène les philosophes, que ce soient les célèbres anciens ou les sophistes qui furent ses contemporains, renvoient évidemment à l'admiration de Lucien pour la Comédie.

61 Le narrateur/auteur de la Mort de Pérégrinos qui fait intervenir le rieur Démocrite à plusieurs reprises dans le texte nous induit, me semble-il, à passer avec Démocrite au « rire avec » particulièrement dans les dernières lignes.

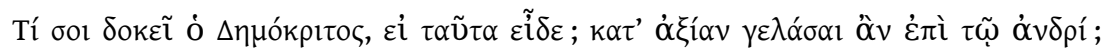

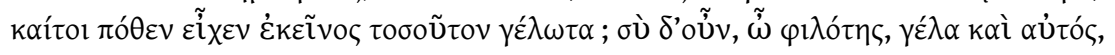

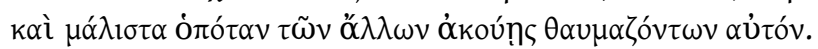

«À ton avis, que ferait Démocrite, s'il avait vu ce spectacle ? Il rirait de cet homme, à juste titre. Cependant d'où pouvait-il, ce philosophe, tirer un aussi grand rire? Toi, en tout cas, mon doux ami, ris bien, toi aussi, et surtout quand tu entendras ses admirateurs ! » (La mort de Pérégrinos 45) 


\section{BIBLIOGRAPHIE}

ANDERSON G., Lucian Theme and variations in the Second Sophistic, Leiden, Brill, 1996.

BALLABRIGA A., « Approches du burlesque divin dans l'antiquité », in Le rire des Grecs, Anthropologie du rire en Grèce ancienne, Desclos M. -L. (dir.), Grenoble, 2000, p. 123-131.

Billault A., " Une 'vie de sophiste' : le Pseudologiste », in Lucien de Samosate, Actes du colloque international de Lyon (1993), Billault A. (dir.), Lyon, 1994, p. 117-124.

BOMPAIRE J., « L'atticisme de Lucien », in Lucien de Samosate, Actes du colloque international de Lyon (1993), Billault A. (dir.), Lyon, 1994, p. 65-75.

BOMPAIRE J., Lucien écrivain, Paris, 1968 (rééd. 2000).

BRANHAM B.R., Unruly Eloquence: Lucian and the Comedy of Traditions, Cambridge/London, 1989.

BRIAND M., « Le dialogue entre mythe et fiction : à propos du Dionysos de Lucien » in Mythe et fiction, D. Auger et Ch. Delattre (dir.), Paris, 2010, p. 217-237.

CAMPOS DAROCA F. J., " Maxime de Tyr, Socrate et les discours selon la philosophie ", in Maxime de Tyr, entre rhétorique et philosophie au II ${ }^{e}$ siècle de notre ère, F. Fauquier et B. Pérez-Jean (dir.), Montpellier, 2016, p. 67-84.

CARRIÈRE J.-Cl., « Socratisme, platonisme et comédie dans le Banquet de Xénophon. Le Banquet de Xénophon et le Phèdre de Platon ", in Le rire des Anciens, Actes du colloque international de Rouen et Paris, M. Trédé et Ph. Hoffmann Ph. (dir.), Paris, 1998, p. 243-271.

DESCLOS M.-L. (dir.), Le rire des Grecs, Anthropologie du rire en Grèce ancienne, Grenoble, 2000.

DESTRÉE P., « Platon et l'ironie dramatique », Revue de métaphysique et de morale, 2013/4, n 80, p. 543-556.

DUBEL S., « Dialogue et autoportrait : les masques de Lucien », in Lucien de Samosate, Actes du colloque international de Lyon (1993), A. Billault (dir.), Lyon, 1994, p. 19-26.

FAUQUIER F. ET PÉREZ-JEAN B., Maxime de Tyr entre rhétorique et philosophie au II siècle de notre ère, Montpellier, PULM, 2016.

FOUCAUlT M., Le courage de la vérité. Le gouvernement de soi et des autres II. Cours au Collège de France (1984), EHESS, Paris, 2009.

FOWLER R., « The second sophistic », in The Cambridge History of Philosophy in Late Antiquity, P. Gerson Lloyd (dir.), Cambridge, 2010.

FUENTÈs GONZALEZ P.P., « Lucien de Samosate », DphA IV, Paris, 2005, p. 131-160.

GEORGIADOU A., LARMOUR David H.G., « Lucian and Historiography ‘De Historia conscribenda' and 'Verae Historiae' «, ANRW 34, 2, 1994, p. 1448-1509.

GEORGIADOU A., LARMOUR David H.G., Lucian's Science Fiction Novel True Histories : Interpretation and Commentary, Leiden, Brill, 1998.

GOULET-CAZÉ M.-O., GOULET R., Le cynisme ancien et ses prolongements. Actes du colloque international du C. N. R. S. (Paris, 22-25 juillet 1991), Paris, Presses Universitaires de France, 1993. 
GOULET-CAZÉ M.-O., «Religion and the Early Cynics », in The Cynics. The Cynic Movement in Antiquity and Its Legacy, R. Bracht Branham et M.-O. Goulet-Cazé (dir.), Berkeley/Los Angeles/London, 1996, p. $47-80$.

GOULET-CAZÉ M.-O., « Ménippe de Gadara », DphA IV, Paris, 2005, p. 467-475.

HALLIWELL S., Greek Laughter. A study of cultural psychology from Homer to early Christianity,

Cambridge, Cambridge University Press, 2008.

HANKINSON R. J., The Sceptics, Londres/New York, Routledge, 1995.

JOUAN F., " Mythe, histoire et philosophie dans les 'Dialogues des Morts' " , in Lucien de Samosate, Actes du colloque international de Lyon (1993), A. Billault (dir.), Lyon, 1994, p. 27-35.

JOUËT-PASTRÉ E., « Le rire chez Platon : un détour sur la voie de la vérité » in M. Trédé et $\mathrm{Ph}$. Hoffmann (dir.), Le rire des Anciens, Actes du colloque international de Rouen et Paris, Paris, 1998, p. 273-279.

LAKS A., MOST G. W., Les débuts de la philosophie, Des premiers penseurs grecs à Socrate, Paris, Fayard, 2016.

LAUWERS J., «The rhetoric of pedagogical narcissism: Philosophy, philotimia and self-display in Maximus of Tyre's first oration ", Classical Quarterly N.s. 59, 2009, p. 593-607.

LAUWERS J., Self-presentation and authority in the Second Sophistic: The case of Maximus of Tyre, Thèse soutenue à l'Université de Louvain, 2012.

MACLEOD, M.D. « Lucianic Studies since 1930 », ANRW II.34.2, 1994 pp. 1362-1421.

MESTRE F., 2013, « Lucien, les philosophes et les philosophies », Ítaca. Quaderns Catalans de Cultura Clàssica, Societat Catalana d'Estudis Clàssics Núm. 28-29 (2012-2013), p. 63-82.

NARCY M., « Le comique, l'ironie, Socrate », in Le rire des Grecs, Anthropologie du rire en Grèce ancienne, M. -L. Desclos (dir.), Grenoble, 2000, p. 282-292.

NESSELRATH H.G., « Lukianos von Samosata », Neue Pauly, Supplemente I Online - Band 7, p . 493-501.

NESSELRATH H.G., « Lucien et le cynisme », L’Antiquité Classique, 67, 1998, p. 121-135.

NIEHUES PRÖBSTING H., Der Kynismus des Diogenes und der Begriff des Zynismus, Frankfurt $1988\left(1^{\mathrm{e}} \mathrm{ed}\right.$. 1979).

NIEHUES PRÖBSTING H., « The modern reception of Cynicism: Diogenes in the Enlightment ", in The Cynics. The Cynic Movement in Antiquity and Its Legacy, R. Bracht Branham et M.-O. Goulet-Cazé (dir.), Berkeley/Los Angeles/London, 1996, p. 329-365.

NI-MHEALLAIGH K., Reading fiction with Lucian: fakes, freaks and hyperreality, Cambridge, Cambridge University Press, 2014

PELLIZER E., « Formes du rire en Grèce antique », in Le rire des Grecs, Anthropologie du rire en Grèce ancienne, M. -L. Desclos (dir.), Grenoble, 2000, p. 43-55.

ROMERI L., Philosophes entre mots et mets, Plutarque, Lucien et Athénée autour de la table de Platon, Grenoble, 2002.

SLOTERDIJK P., Critique de la raison cynique, H. Hildenbrand (trad.) Paris, Christian Bourgois, $1987\left(1^{\mathrm{e}}\right.$ éd. Allemande 1983). 
SOMMERSTEIN A., « Parler du rire chez Aristophane », in Le rire des Grecs, Anthropologie du rire en Grèce ancienne, M. -L. Desclos (dir.), Grenoble, 2000, p. 65-75.

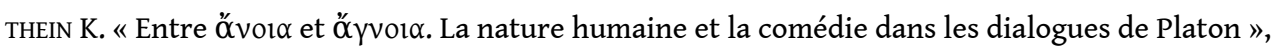
in Le rire des Grecs, Anthropologie du rire en Grèce ancienne, M. -L. Desclos (dir.), Grenoble, 2000, p. 169-180.

TRAPP M. B., « Plato's Phaedrus in the Second Sophistic », in Antonine Literature, D.A. Russell (dir.), Oxford, 1990, p. 141-173.

WHITMARSH T., Greek Literature and the Roman Empire, The politics of imitation, Oxford, 2001.

\section{NOTES}

1. Comme le fait par exemple l'ouvrage récent de Karen NI-MHEALLAIGH, Reading fiction with Lucian : fakes, freaks and hyperreality, Cambridge, Cambridge University Press, 2014.

2. Michel BRIAND, «Le dialogue entre mythe et fiction : à propos du Dionysos de Lucien » in Auger D. et Ch. Delattre (dir.), Mythe et fiction, PUPO, 2010, p. 217 -237.

3. On peut étudier chez Lucien les « intertextualités internes» (ce que fait par exemple Michel Briand avec la prolalie Dionysos), mais bien sûr d'autres commentateurs ont recherché les rapports d'intertextualité avec Arrien, Diodore de Sicile, Nonnos de Pannopolis etc. L'appétit dans la recherche des sources conduit même à poursuivre les arrière-plans du Prométhée de Lucien non seulement chez Homère et Hésiode mais jusque dans la mythologie mésopotamienne. De manière générale, de grands débats ont opposé les spécialistes au siècle dernier sur la question des sources de Lucien et de son originalité. Ainsi les remarques de Macleod (MACLEOD, 1994 :1364-66) sur le livre de J. Bompaire (BOMPAIRE, 1968) qu'il résume avec la citation de Musset : «Pourquoi désavouer l'imitation, si elle est belle?». Il considère que Bompaire insiste trop sur la mimesis et sur la création rhétorique de Lucien au détriment de sa création littéraire et de sa propre étude sur la fantaisie de Lucien dont il aurait sous estimé l'originalité.

Or une nouvelle dimension de l'intertextualité se fait jour dans les recherches sur la seconde sophistique et particulièrement sur Lucien à partir d'une série d'articles italiens ou anglais rapprochant les « romans » ou nouvelles de Lucien, en particulier l'Onos (son attribution à Lucien est toujours discutée) et le Nom de la Rose d'Umberto Eco. Ces travaux prennent ainsi leur point de départ dans ceux d'Eco lui-même sur le post-modernisme qui n'est pas un style attaché à un moment historique et culturel particulier, mais une attitude qui peut sous-tendre toute production dans toute ère culturelle, une catégorie idéale, un Kunstwollen, une manière d'opérer en art littéraire, au point de soutenir que toute période a son propre post modernisme; en ce sens Whitmarsh (WHITMARSH, 2001) trouve des affinités entre la culture du spectaculaire de la Rome impériale et le postmodernisme actuel. K. Ni-Mheallaigh (NI-MHEALLAIGH, $2014: 24$ ) reprend à Eco la notion d'hyperréalité dans son analyse du pseudos chez Lucien.

4. Bien des critères sont utilisés pour tenter de cerner le mouvement de la seconde sophistique et le milieu intellectuel qui est celui de Lucien: conception agonistique de la performance, construction d'une opposition stylistique entre atticisme et asianisme, posture dans la performance, apparence vestimentaire, positionnement dans la hiérarchie des genres sexuels, dans l'originalité sociale de l'élite, recherche de l'expression du soi, autobiographie, construction du moi doloriste chez Aelius Aristide, recherche d'une cohésion culturelle avec l'idéologie impériale, analyse de la distinction au sens bourdieusien, opposition entre nature et culture, entre paideia urbaine et sagesse naturelle, valorisation simultanée du savoir académique et de la spontanéité du discours ex tempore, etc. 
5. La danse tente de redonner du prestige à la pantomime qui fut un art populaire important dans le monde romain mais se trouvait déclassé à cette époque (NI-MHEALLAIGH, 2014 :18).

6. «C'est une forme de la vanité du vingt et unième siècle de prétendre que notre temps a été le premier dans l'histoire humaine à avoir exprimé et exploré ses propres crises idéologiques et épistémologiques à travers le spectaculaire » (WHITMARSH, 2001 : 254, cité par NI-MHEALLAIGH, 2014 : 107).

7. Malheureusement la critique peine à donner une chronologie précise. On peut considérer que dans la préface de $H V$ l'allusion aux ouvrages plus sérieux (spoudaioteros) est une référence à son autre œuvre.

8. Ces auteurs développent leur article dans leur ouvrage: Lucian's Science Fiction Novel True Histories : Interpretation and Commentary, Brill, 1998.

9. Sur les nombreux cas de « cross-referentiality » dans de nombreux opuscules de Lucien voir NIMHEALLAIGH, 2014, passim.

10. C'est ce qu'on a pu dire du rire socratique ; voir JOUET-PASTRÉ, 1998 : 279.

11. À propos d'Alexandre ou le faux prophète, Branham a étudié les rapports entre la narration et le genre de la wonder literature et a montré l'importance de ce type de sources dans la fantaisie de Lucien.

On rappellera le terme de kenodoxia bien connu par le fameux prologue de $H V$ et l'attribution par Lucien à l'archi-menteur Ulysse de bômolochia et alazoneia (bouffonnerie et charlatanerie).

12. Alain Billault, Une « vie de sophiste » : le Pseudologiste, in A. Billault ed., Lucien de Samosate, Actes du colloque international de Lyon (1993), Lyon, 1994, p. 117-124.

13. A. Billault taxe Lucien «d'acharnement de polémiste » et de «tempérament porté à détruire qui lui a déplu ».

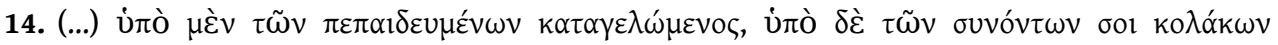

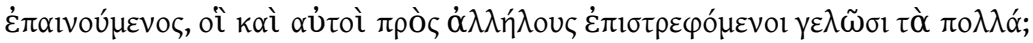

(...) moqué par les gens cultivés et loué par les flatteurs qui te fréquentent, qui eux aussi se tournent les uns vers les autres en riant tout leur soûl? (Adversus indoctum et libros multos ementem, 7).

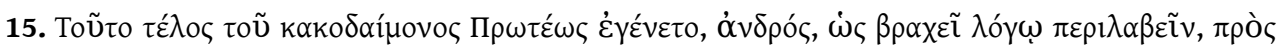

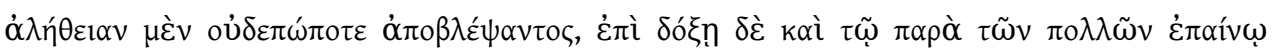

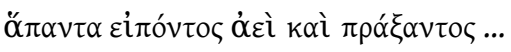

Tel fut donc le destin de ce misérable Protée, un homme, pour le dire en peu de mots, qui n'a jamais tourné ses regards vers la vérité mais a toujours soumis ses propos et ses actes à la gloriole et à l'éloge général. (De morte Peregrini, 42).

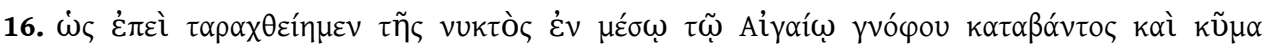

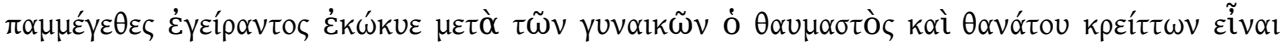

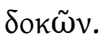

17. D. L. IX, 68 Pyrrhon loue l'ataraxie du cochon pendant la tempête et la donne en exemple aux marins.

18. Il existe bien des parallèles entre Dion et Lucien. Voir par exemple PERNOT, in BILLAULT 1994.

19. Cf. aussi l'introduction de Philippe Renault à ses traductions sur BCS.

20. Sur ce débat, voir MESTRE F., 2013, "Lucien, les philosophes et les philosophies ", Ítaca. Quaderns Catalans de Cultura Clàssica, Societat Catalana d'Estudis Clàssics Núm. 28-29 (2012-2013), p. 63-82, en particulier p. 79.

21. SLOTERDIJK Peter, Critique de la raison cynique, traduction française par H. Hildenbrand de l'édition allemande de 1983, Paris, Christian Bourgois, 1987.

22. Voire le christianisme (SLOTERDIJK, $1987: 220$ ).

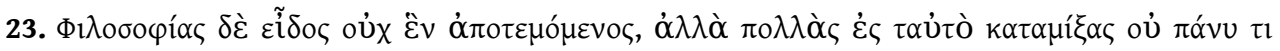

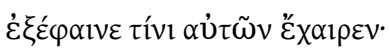


24. Sur les liens entre Banquet de Xénophon, Phèdre et Banquet de Platon et Banquet de Lucien voir J.-Claude Carrière (CARRIÈRE, 1998), R. Bracht Branham (BRANHAM, 1989) et surtout Luciana Romeri ( ROMERI, 2002) et sa thèse du renversement dans une version différente de l'inversion bakhtinienne de Branham.

25. Le débat Anderson /Macleod porte sur la profondeur de la culture littéraire et philosophique de Lucien : ne possède-t-il qu'un stock de citations?

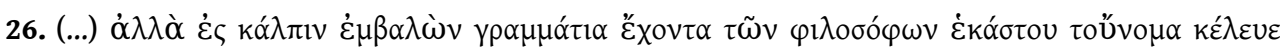

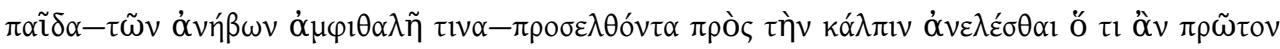
ن்

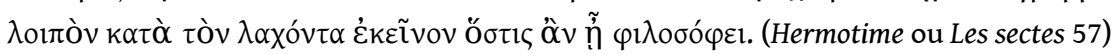

(...) mais mets dans une urne des tablettes portant le nom de chacun des philosophes et dis à un jeune enfant qui a encore son père et sa mère de s'approcher de l'urne et d'en tirer la première tablette qui lui tombera sous la main, et quel que soit le nom du philosophe que le hasard apportera, mets toi désormais à philosopher selon ses préceptes. (trad. E . Chambry modifiée)

27. АГОРАГТН

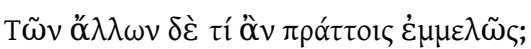

MYPPSN

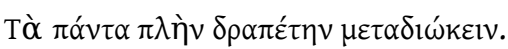

АГОРАГТН

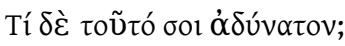

$\Pi Y P P \Omega N$

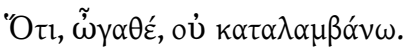

L'acheteur : Pour le reste que peux-tu faire convenablement?

Pyrrhon : Tout sauf poursuivre un esclave fugitif

Acheur : Mais pourquoi cela t'est-il impossible?

Pyrrhon : Parce que je ne peux saisir, mon bon! (Vitarum auctio, 27)

(Cf. Sextus Empiricus HP I, les formules sceptiques, par exemple : o= Sextus Empiricus HP I, on bon!saisir mon bon, et plsu de 20àans pour istique. et vérité;elon Sloterdijk en tant que cukltivé eủ $\kappa \alpha \tau \alpha \lambda \alpha \mu \beta \alpha ́ \alpha \omega \omega)$.

HANKINSON $1995: 150$ rapproche cette scène des critiques de Galien.

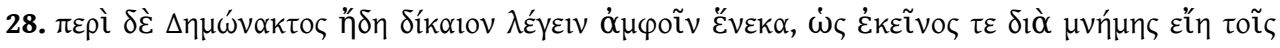

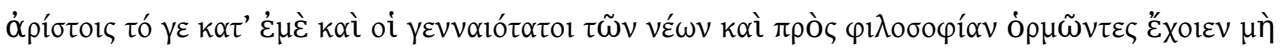

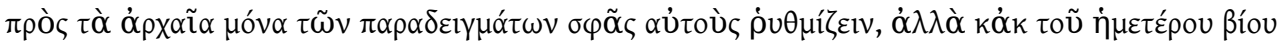

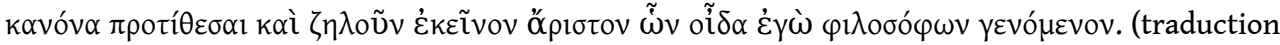
Bompaire modifiée).

29. Voir la contribution F. JOUAN in BILLAULT 1994 : 27-35.

30. Ce que fait par exemple Alain MICHEL in BILLAULt 1994.

31. On pense aux études classiques de Chambry, Caster, Bompaire témoignant de l'importance de son athéisme dans la tradition française, du XVI ${ }^{\mathrm{e}}$ au XVIII ${ }^{\mathrm{e}}$ siècle.

32. Sa description sinistre et détaillée des Enfers, écrit Ph. Renault dans Bibliotheca classica selecta (http ://bcs.fltr.ucl.ac.be), est savoureuse à souhait, et son goût pour le macabre et l'excessif, en même temps que ses notations bouffonnes proprement irrésistibles, confirment une imagination débordante et une verve corrosive. Certes, comme toujours chez Lucien, on trouve ici ou là des références littéraires nombreuses, puisées chez Homère ou chez les Tragiques ; mais si les grands auteurs sont cités, c'est pour mieux les parodier.

Encore une fois - c'est le leitmotiv de la plupart de ses dialogues -, Lucien dénonce les philosophes, sa bête noire, ne manquant pas de les discréditer et de les ridiculiser par la voix de Ménippe qui, on le devine, doit être identifié avec notre auteur. Seul Tirésias est un sage et détient la bonne réponse qui satisfera l'homme de Gadara venu spécialement le consulter : cette 
réponse est en accord avec les grands principes de la doctrine cynique, même si elle ne le dit pas. En effet, l'appel de Tirésias à profiter de l'instant, et son hostilité à vouloir expliquer les choses célestes qui nous dépassent, résument admirablement la pensée cynique, dont Lucien est un adepte, même s'il la mâtine généralement avec les idées épicuriennes qui ont également sa prédilection.

33. TIMOK $\Lambda \mathrm{L}$

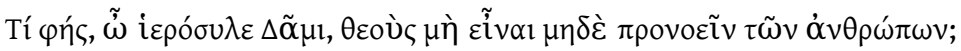
$\triangle \mathrm{AMI \Sigma}$

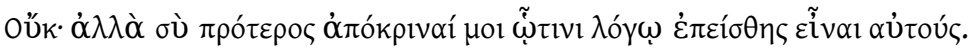

Pourquoi dis-tu, sacrilège Damis, que les dieux n'existent pas et n'exercent pas leur providence envers les hommes?

Damis : Non ils n'existent pas mais réponds moi d'abord toi : pour quelle raison crois-tu qu'ils existent? (Zeus tragique, 35)

Le débat qui fait intervenir Zeus, Hermès et Momos se poursuit jusqu'à la fin du dialogue où Damis fait mine d'abandonner sa critique des dieux puisque Timoclès se réfugie près de leurs autels. Zeus conclut en parodiant une parole de Darius à propos de Zopyre chez Hérodote : «j'aimerais mieux moi aussi avoir pour allié le seul Damis que de posséder dix mille Babylones » (Zeus tragique, 50).

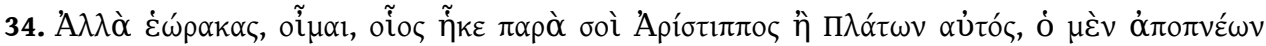

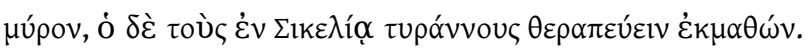

Mais tu as vu, je pense, dans quel état sont arrivés près de toi Aristippe et Platon même, l'un fleurait les parfums et l'autre avait appris à fond en Sicile à flatter les tyrans. (Dialogues des Morts $6,5)$.

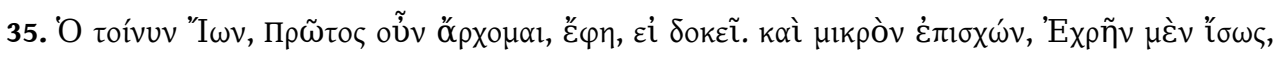

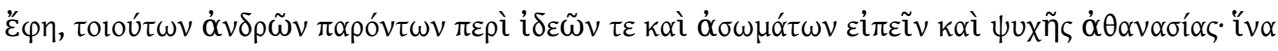

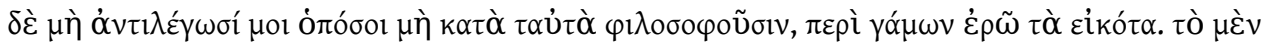

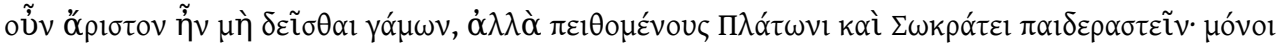

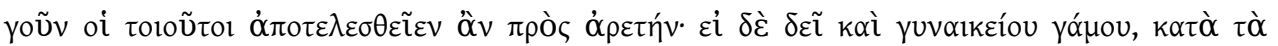

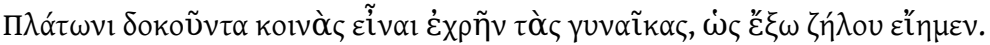

Ion prit la parole: "Je parle donc en premier, si bon vous semble ». Et après une légère suspension: «Il faudrait peut-être, dit-il, en présence d'hommes d'un telle qualité parler des idées et des incorporels et aussi de l'immortalité de l'âme ; mais afin que ne me contredisent pas tous ceux qui ne philosophent pas selon ces principes, j'exposerai ce qui est vraisemblable à propos du mariage. Donc la meilleure chose à faire serait de ne pas se marier, mais en suivant Platon et Socrate de choisir l'amour des garçons; il est certain que seuls ceux qui font un tel choix seront capables d'atteindre la vertu. Mais s'il faut recourir au mariage avec des femmes, il faudrait selon l'opinion de Platon, qu'elles soient mises en commun afin que nous soyons exempts de jalousie. (Symposium, 39).

36. Selon J. Campos Daroca les enjeux de la controverse socratique avaient évolué de la manière suivante : a) Socrate est devenu le fondateur de la philosophie morale, diversifiée ensuite selon plusieurs écoles et, de plus, une étape disputée dans des «successions » et " généalogies » de la philosophie; b) les formes diverses de l'enseignement socratique ont été réinterprétées et sujettes à des versions rivales, aussi bien rhétoriques que philosophiques; c) la vie de Socrate a donné naissance à des traitements biographiques d'orientations diverses et, en même temps, certains moments de son activité ont acquis la condition d'images exemplaires de la philosophie ; et $\mathrm{d}$ ) on commence à faire des différences entre les «sources" sur Socrate, où s'amorcent des distinctions entre les divers auteurs « socratiques".

37. Dans la première des Dialéxeis dédiées à l'art amoureux de Socrate (XVIII), on trouve une interprétation de l'activité socratique très proche de celle que l'on peut lire chez Dion Sur Homère et Socrate. Ils partageaient les mêmes inquiétudes pédagogiques, puisque tous les deux se 
souciaient de la vertu et du vice, de la vérité et du mensonge, des opinions des sots et des connaissances des hommes sages.

Maxime paraît faire face à de nouveaux accusateurs de Socrate, scandalisés, à ce qu'il paraît, par ses propos amoureux et les leçons que l'on pourrait tirer d'un sujet double comme celui de l'amour, où un seul mot signifie deux choses moralement opposées (\$ 4). En conséquence, Maxime se propose d'examiner avec l'attention qu'elle mérite la manière tout à fait scandaleuse que Socrate avait de parler de l'amour, conscient des périls qui peuvent en surgir, si cette passion n'est pas bien comprise.

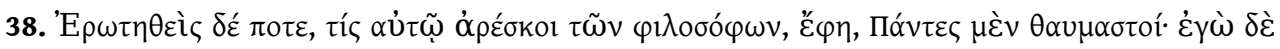

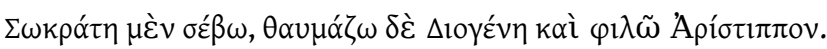

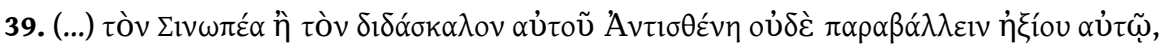

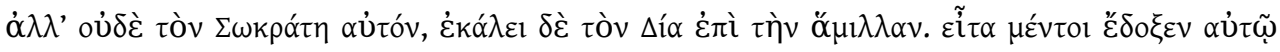

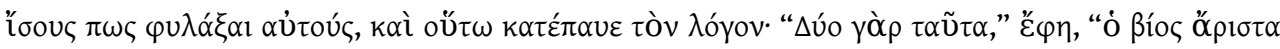

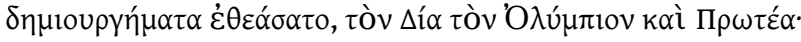

(...) le philosophe de Sinope ni son maître Antisthène ne méritaient de rivaliser avec lui, mais pas même Socrate, et c'est Zeus qu'il appelait à la confrontation. Ensuite il crut bon de les mettre à égalité et ainsi, il termina sur ces mots : « Il y a deux entités que la vie a contemplées comme les plus grandes créations, Zeus Olympien et Protée ». (De morte Peregrini, 5).

40. Un sophiste du $\mathrm{II}^{\mathrm{e}}$ ou du $\mathrm{III}^{\mathrm{e}}$ siècle était avant tout connu pour son style recherché et fleuri ; un philosophe, à cette époque, pouvait admettre le titre de sophiste et, preuve du renversement de perspective par rapport à l'ancienne Sophistique que Platon et Aristote avaient réduite au pseudos (non-être, faux, falsification), selon Philostrate, seuls les meilleurs philosophes peuvent accéder au nom et au statut de sophistes. Le philosophe peut parfois rejeter le nom de sophiste, mais la réputation d'éloquence est essentielle pour le professeur. De fait, le terme de sophiste aux $\mathrm{II}^{\mathrm{e}}$ et $\mathrm{III}^{\mathrm{e}}$ siècles désigne aussi ce type de professeurs ou de conférenciers itinérants. Les philosophes deviennent ainsi des artistes qui donnent des performances dans «tournées philosophiques » comme Dion de Pruse, Favorinus d'Arles et Lucien lui-même. La popularité des déclamations rhétoriques, très importante de Plutarque à Plotin, chez les Platoniciens en particulier, décroit peu à peu entre le $\mathrm{II}^{\mathrm{e}}$ et le $\mathrm{V}^{\mathrm{e}}$ siècles. Mais à l'époque de ou de Lucien, de Maxime de Tyr, de Dion de Pruse, elle est à son apogée. (FAUQUIER, PÉREZ-JEAN, 2016 :13-16).

41. Cette parrêsia est «liée à un certain mode de vie » et il rapproche la liberté de parole que pouvait se permettre Socrate dans le Lachès à l'égard des honorables Nicias et Lachès, ce " courage de la vérité » auquel Socrate « s'autorisait au fond dans la mesure où il avait donné dans sa vie un certain nombre de gages et de garanties ». "Le rapport entre dire vrai et mode de vie chez les cyniques entre bien dans le cadre général de cette homophonie entre le dire et le vivre qui était désignée dans le Lachès ». Mais, bien sûr, la grande différence avec le socratisme est que dans le cynisme "ce n'est pas simplement la vie qui manifeste un certain nombre de vertus ", comme Socrate avec tempérance courage sagesse... (FOUCAULT, $2009: 157 ; 184$ ).

42. Foucault commente en particulier la comparaison entre le procès de Socrate et le procès d'impiété auquel Lucien dit que vient d'être confronté Démonax avec les deux chefs d'accusation : ne pas avoir sacrifié à Athéna et avoir refusé d'être initié aux Mystères d'éleusis. L'amour de l'humanité (philanthrôpia) de Démonax supposait une parrêsia, une liberté de parole impliquant qu'il révèle toute vérité possible à propos des Mystères. "Il ne voulait donc pas se faire initier lui même car, s'il avait été initié, il aurait été obligé de s'engager à se taire». (FOUCAULT, 2009 : 156).

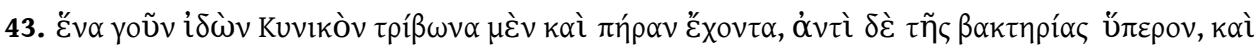

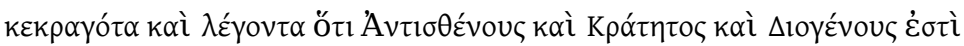

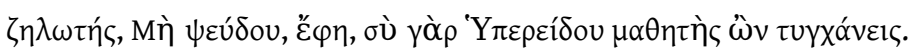

Voyant un cynique qui portait le manteau et la besace et à la place du bâton une massue 
(hyperon), qui criait et disait qu'il était un disciple d'Antisthène, Cratès et Diogène, $<$ Démonax > lui dit : « Ne mens pas, toi, tu es un élève d'Hypéride » (Vie de Démonax 48).

44. La référence de son autocitation est en note 44 p. 75.

45. Voici le résumé de Philippe Renault (http ://bcs.fltr.ucl.ac.be/LUCIEN/Mort.html) :

Pérégrinos fut emprisonné pour sa foi mais vite libéré par une administration romaine plutôt tolérante. Rejeté ensuite par les Chrétiens pour avoir manqué à leurs rites - il avait mangé une viande proscrite -, il revint au cynisme et se fit brûler très solennellement aux jeux olympiques de 165 , immolation qui ne manque pas de nous rappeler le rituel sacrificiel identique pratiqué par Calanos à Suse en présence d'Alexandre le Grand et par Zarmaros à Athènes, au temps d'Auguste. Écrivant peu après l'événement, ce qui permet de dater clairement son récit (165 ou 166), Lucien tient à rétablir la vérité sur ce philosophe qu'il considère comme un imposteur notoire et dont l'ardeur de la foi était à ses yeux totalement feinte.

Cependant, des critiques modernes tels que Zeller, Wilamowitz et plus près de nous Caster, font de lui un mystique sincère, soucieux de vérité, dont le suicide final justifie la valeur mystique de la quête. Beaucoup d'entre eux s'appuient sur le témoignage d'Aulu-Gelle qui brosse dans ses Nuits attiques [XII, 11] un portrait positif de Pérégrinos, « doux dingue » certes, mais aussi homme intègre. La soif d'honneurs et de gloire, bref la vanité, auraient été exagérées par un Lucien, trop haineux à son égard, n'ayant en rien compris la profondeur spirituelle de ce chaman.

\section{RÉSUMÉS}

Trois termes, mythe, histoire et fiction, doivent être mis en relation à propos de la seconde sophistique et particulièrement à propos de Lucien dont les textes se focalisent de manière obsessionnelle sur la question de la réception de la littérature et de la production artistique, par exemple dans la relation entre Histoires vraies $(\mathrm{HV})$ et De historia conscribenda, à propos la question de savoir comment écrire l'histoire dans l'Antiquité. Un trait fondamental de l'œuvre de Lucien est le plaisir paradoxal du pseudos. On caractérisera l'auteur Lucien, à la suite des travaux de Robert Bracht Branham concernant la différence entre parodie et satire, par la formule de spoudogeloios, serio-comic. Le rire du lecteur et le rire des personnages, voire de Lucien ou de son double, sont analysés ici particulièrement en prenant comme cibles les philosophes, et comme sujets de satire voire de parodie leurs prétentions non à la détention de la vérité mais à la célébrité ou bien l'écart entre les paroles et les actes.

Dans Vitarum auctio, la parodie met en scène la connaissance précise des systèmes philosophiques mis à l'étalage et porte sur l'écart entre la précision scientifique exprimée avec les termes techniques appropriés par le crieur et l'incapacité de l'acheteur à se représenter de quoi il peut s'agir ainsi que l'utilité didactique qu'il pourrait retirer de son éventuel achat. En se démarquant de la lecture de Sloterdijk qui permet certes de considérer Lucien comme un véritable philosophe, mais fait de lui un cynique opportuniste et méprisant, l'étude recherche l'orientation philosophique de Lucien et conclut à partir de ses biographies de Pérégrinos et de Démonax sur la nature de son cynisme et de son rire.

Three terms, myth, history and fiction, are to be connected about the Second Sophistic and especially Lucian whose works focus in a obsessive manner upon the reception of literature and artistic production: see for instance the links between True Histories and De historia conscribenda. The main feature in Lucian's works is the paradoxical pleasure of the pseudos. 
Robert Bracht Branham studies, especially about parody and satire in Lucian, bring us to call the author spoudogeloios, serio-comic. This study analyses the laughter of the reader, of the characters, of Lucian himself or his double about philosophers. The fact that they seek not to know the truth but to be famous and the gap between speeches and deeds are the main topics of the satire or even the parody. In vitarum auctio, parody shows the precise knowledge of philosophic systems sold at the auction and the gap between the scientific exactness of the philosophical terms used by the seller and the inability of the byer to imagine what didactic use he could make with this purchase. Contrasting with Sloterdijk's interpretation which of course allows to see Lucian as a true philosopher but shows him as an opportunistic and dispising cynic, this study looks for the Lucian's philosophical orientation and comparing Peregrinos' and Demonax' biographies depicts his kind of cynicism and laughter.

INDEX

Mots-clés : Lucien, philosophe, parodie, cynique, rire

Keywords : Lucian, Philosopher, Parody, Cynic, Laughter

\section{AUTEUR}

\section{BRIGITTE PÉREZ-JEAN}

Brigitte Pérez-Jean, Professeur de Langue et littérature grecques à l'Université Paul-Valéry, Montpellier 3, E.A. 4424 C.R.I.S.E.S a publié différents articles et ouvrages (études, traductions, actes de colloque) dans le domaine de la philosophie grecque antique. Dernière publication : Maxime de Tyr entre rhétorique et philosophie au IIe siècle de notre ère, Fr. Fauquier et $\mathrm{Br}$. Pérez-Jean éd., PULM 2016. 